\title{
Altered visual perception near the hands: A critical review of attentional and neurophysiological models
}

\author{
Stephanie C. Goodhew ${ }^{1}$, Mark Edwards ${ }^{1}$, Susanne Ferber ${ }^{2}$, and Jay Pratt $^{2}$ \\ ${ }^{1}$ Research School of Psychology, The Australian National University \\ ${ }^{2}$ Department of Psychology, The University of Toronto
}

Word count: (main text): 9,401

Corresponding Author: Stephanie C. Goodhew

Address: Research School of Psychology (Building 39)

The Australian National University, Canberra, 0200

Email: stephanie.goodhew@anu.edu.au

Running head: Visual perception near the hands 


\begin{abstract}
Visual perception changes as a function of hand proximity. While various theoretical accounts have been offered for this alteration (attentional prioritization, bimodal cell involvement, detailed evaluation, and magnocellular neuron input enhancement), the current literature lacks consensus on these mechanisms. The purpose of this review, therefore, is to critically review the existing body of literature in light of these distinct theoretical accounts. We find that a growing number of results support the magnocellular (M-cell) enhancement account, and are difficult to reconcile with general attention-based explanations. Despite this key theoretical development in the field, there has been some ambiguity with interpretations offered in recent papers, for example, equating the existing attentional and M-cell based explanations, when in fact they make contrasting predictions. We therefore highlight the differential predictions arising from the distinct theoretical accounts. Importantly, however, we also offer novel perspectives that synthesises the role of attention and neurophysiological mechanisms in understanding altered visual perception near the hands. We envisage that this theoretical development will ensure that the field can progress from documenting behavioural differences, to a consensus on the underlying visual and neurophysiological mechanisms.
\end{abstract}

Keywords: near-hand space; peripersonal space; attention; attentional spotlight; magnocellular; parvocellular; spatial acuity; temporal acuity; bimodal neurons; bimodal cells 


\section{Near-hand space}

A body of literature indicates that visual perception and performance is altered when visual stimuli occur in the space near the hands (see Brockmole, Davoli, Abrams, \& Witt, 2013; for a review). That is, identical visual information is processed differently dependent on the relationship between the visual information and the observer's hands. The typical laboratory set-up in which near versus far-hand space is manipulated is to have observers place their hands on response-equipment attached to either side of the screen, rendering the visual stimuli displayed on the computer screen in "near-hand space", versus having the observer place their hands on response-equipment in their lap, or on the desk in front of them, rendering the visual stimuli on the screen in "far-hand space". A plethora of evidence suggests that such near versus far-hand set-up produces differences in performance on a variety of visual tasks (Abrams, Davoli, Du, Knapp, \& Paull, 2008; Adam, Bovend'Eerdt, van Dooren, Fischer, \& Pratt, 2012; Chan, Peterson, Barense, \& Pratt, 2013; Cosman \& Vecera, 2010; Davoli, Du, Montana, Gaverick, \& Abrams, 2010; Garza, Strom, Wright, Roberts, \& Reed, 2013; Goodhew, Fogel, \& Pratt, 2014; Goodhew, Gozli, Ferber, \& Pratt, 2013a; Gozli, Ardron, \& Pratt, 2014; Gozli, West, \& Pratt, 2012; Tseng \& Bridgeman, 2011). Importantly, this altered perception is attributable to the hand proximity, rather than hand visibility or response mode, as similar effects occur even when the observer's hands are concealed from their view, and when they use their feet rather than their hands to respond (Abrams et al., 2008; Reed, Grubb, \& Steele, 2006). Furthermore, it is hand proximity, rather than posture, that creates these effects (Weidler \& Abrams, 2013). Thus, altered visual perception reflects the effect of hand proximity, rather than some other variable, most likely due to a mechanism that registers combined proprioceptive and visual input.

Given the body of evidence for altered visual perception in near-hand space, a key question pertains to the nature and therefore mechanism underlying this difference. A number 
of different theoretical accounts have been put forward. Initial accounts drew on visual attention as an explanation. However, as this review will discuss, there is some ambiguity and a lack of precision about the use of 'visual attention' to explain quite a diverse and at times even apparently contradictory pattern of findings. A more recent account draws on more precise physiological underpinnings, specifically, the interplay of the two major classes of visual cells, magnocellular neurons (M-cells) and parvocellular neurons (P-cells). This account has the advantage of making clearer, more precise predictions, and evidence is accumulating that this account can explain elegantly what the general attentional accounts struggle to account for. The purpose of this review is to highlight this crucial theoretical development, because there remains a tendency to rely on the earlier, less-developed theoretical accounts to explain findings, with either no reference to the more recent theories or the erroneous assumption that they are different ways of describing the same theory (e.g., Park, Strom, \& Reed, 2013; Reed, Leland, Brekke, \& Hartley, 2013). Following this, however, we also offer some new insights into how a more nuanced understanding of visual attention, in particular how different mechanisms of attention relate to underlying neurophysiology, may ultimately unite accounts based on attention versus input biases from magnocellular versus parvocellular neurons.

\section{Visual Attention}

Visual attention is a process in which particular stimuli are selected for privileged processing at the expense of others. This process of selection is thought to be necessary, so as not to overwhelm the brain's limited perceptual and cognitive processing resources (Broadbent, 1958; Desimone \& Duncan, 1995; Kastner \& Pinsk, 2004). This process can operate in a bottom-up or reflexive manner, in which brute stimulus energy or salience determines the stimuli that are selected (Jonides \& Yantis, 1988; Yantis \& Jonides, 1984), or it can operate in a top-down or more strategic way, in which an observer's goals guide 
selection (e.g., Becker, Folk, \& Remington, 2010; Folk, Remington, \& Johnston, 1992; Goodhew, Kendall, Ferber, \& Pratt, 2014; Most et al., 2001; Wyble, Folk, \& Potter, 2013).

\section{Attentional Prioritization Theory and Bimodal Cells}

Reed, Grubb, and Steele (2006) offered the first theoretical account for altered visual perception near the hands, proposing that near-hand space enjoys attentional prioritization. This explanation was motivated by their results from a covert attentional orienting paradigm (Posner, Walker, Friedrich, \& Rafal, 1987). In their paradigm, participants' task was to detect the onset of a target - a filled-in square (i.e., a rapid change in luminance at the target location) that could appear at one of two possible locations, which were demarcated with outline squares. A cue, which consisted of the border of one of the two squares darkening, preceded the target. The cue was $70 \%$ valid, which means that the cue correctly signalled the location of the target on $70 \%$ of trials (valid trials) and the target appeared in the uncued location on the other $30 \%$ of trials (invalid trials), and response times (RTs) to detect the target were measured as a function of cue validity. These authors manipulated near versus far hand space in a slightly different manner from most subsequent studies: participants placed a single outstretched hand, with their palm facing toward the centre of the screen and the tips of their fingers touching the screen. This rendered the stimuli on the same side of the screen as their hand in "near-hand space", whereas stimuli appearing on the opposite side of the screen were deemed "far-hand space”. Reed et al. found that participants were faster to respond to targets when they appeared in near-hand space compared with when they appeared in farhand space. Importantly, however, there was no change in cueing (the difference in RT between valid and invalid trials) between near and far-hand space. Since cueing is the hallmark of a shift of spatial attention (Posner, 1980), this indicates that participants' efficiency in shifting their attention from an invalidly cued location to the location of the target was unaffected by hand proximity. Subsequent control experiments eliminated 
explanations for this difference between general accelerated processing without specific attentional effects based on visual anchoring (a board aligned to the screen in place of the hand had no such effect), or hand visibility (the same effects were observed when the hands were concealed from the observer as when they were visible). This led Reed and colleagues to suggest that there is a baseline attentional advantage in near-hand space, unrelated to operations of shifting attention, called the attentional prioritization account (Reed et al., 2006; see also Reed, Betz, Garza, \& Roberts, 2010). To summarise, the attentional prioritization account makes a very clear prediction: facilitation or enhancement for all visual tasks in nearhand space.

Reed et al. (2006, 2010) postulated that bimodal cells are the biological underpinning of attentional prioritization in near-hand space. Bimodal cells are those which respond equivalently to both visual and tactile stimulation falling in their receptive fields. The receptive fields of these cells are hand-centred, meaning that they dynamically update according to the position of the hand in space. These cells have been identified in posterior parietal and premotor cortex in monkeys via single-cell recording (Graziano, Yap, \& Gross, 1994), and more recently identified in humans with functional magnetic resonance imaging (fMRI) (Brozzoli, Gentile, \& Ehrsson, 2012). According to Reed et al. (2006), bimodal cells are important for integrating visual and tactile information for spatial attention. To support this idea, these authors point out that nonpredictive visual cues can facilitate responses to tactile targets presented to the hand, and similarly that tactile cues near the hand facilitate responses to visual targets (Spence, Nicholls, Gillespie, \& Driver, 1998), and this depends on hand proximity not visibility (Kennett, Spence, \& Driver, 2002). Thus, according to Reed et al. (2006, 2010), bimodal cells are the biological basis for attentional prioritization in nearhand space, which predicts enhancement for all visual-cognitive tasks. 


\section{Detailed-Evaluation Theory}

Another attentional account of altered visual processing near the hands was suggested by Abrams et al. (2008), which appears to contradict the predictions from the attentional prioritization account. This theory was motivated by Abrams et al.'s results on three classic attentional tasks: visual search, inhibition of return (IOR) in a cueing task, and the attentional blink (AB). Each of these results is discussed in detail below. These authors used what has become the more conventional hand proximity manipulation, in which observers responded via buttons placed on either side of the computer monitor (near-hand space), or conversely via response buttons on a board in their lap (far-hand space).

Visual search involves search for a target object amongst distractors, time to locate or identify the target is measured. When the target is not distinguished from distractors by a unique feature (e.g., colour), then search is inefficient, and search times can be used to infer processing time for each individual item (Treisman \& Gelade, 1980). In Abrams et al.’s (2008) experiment, the task was to identify one of two possible target options (the letter ' $\mathrm{H}$ ' or ' $S$ ') amongst distractor letters, and the displays consisted of either four or eight items. These authors found that visual search times were slower in near-hand space compared to farhand space. That is, contrary to the attentional prioritization account, participants appeared to be dwelling longer on each letter in the display, slowing their overall search times relative to when the identical stimuli were presented further from the hands. These results did not depend on the participant's hands being visible to them, or on participants making responses with their hands (identical differences dependent on hand proximity even when responding via foot pedals) (Abrams et al., 2008).

In Experiment 2, Abrams et al. (2008) employed a classic attentional cueing paradigm, similar to that used by Reed et al. (2006), except that now the cues were 
uninformative (i.e., not predictive of the subsequent target location). It is well established that at short cue-target intervals, cueing is observed, such that responses are quicker for validlycued compared to invalidly-cued trials. However, this pattern qualitatively shifts with increasing cue-target stimulus-onset asynchrony (SOA), such that responses are slower for validly-cued trials compared to valid, known as IOR (Posner \& Cohen, 1984; Posner, Rafal, Choate, \& Vaughan, 1985). IOR is thought to originate from the process of disengagement and subsequent cost to re-engage attention at a previously-attended location. This likely reflects an adaptive mechanism that facilitates efficient visual search by preventing repetition (Klein, 2000). Abrams et al. (2008) used a 300ms (where cueing is expected) and a 950ms (where IOR is expected) cue to target SOA. As expected, at 300ms SOA, there was cueing, such that validly-cued trials were facilitated in response compared to invalidly-cued trials, however, this advantage was unaffected by hand proximity. At 950ms, IOR was observed, and the magnitude of IOR was reduced for hands-near relative to the hands-far condition. This is consistent with the notion that, similar to the visual search result, participants appeared to be spending longer processing items in near-hand space, delaying their attentional disengagement (Abrams et al., 2008).

In their third experiment, Abrams et al. (2008) used an attentional-blink (AB) paradigm, in which participants' task is to identify two targets in a rapid serial visual presentation stream at a single, central spatial location. The AB refers to the characteristic dip in second target perception when it occurs within a 200-500ms window of the first target (Dux \& Marois, 2009; Raymond, Shapiro, \& Arnell, 1992). The AB can be used as a metric of attentional disengagement, because it is said to reflect the time required to disengage from the first target to make resources available to process the second target (Duncan, Ward, \& Shapiro, 1994; Raymond, et al., 1992), and the AB deficit is directly increased when processing time for the first target is increased (Jolicoeur, 1998; Visser, 2007). Abrams et al. 
(2008) used alphanumeric characters as stimuli: the first target was a digit (2-9), and participants' task was to identify its parity (i.e., odd/even), and the second target was a letter (A/B), and the task was to identify whether it was ' $\mathrm{A}$ ' or ' $\mathrm{B}$ '. Distractor stimuli in the stream were other letters. The AB deficit was exacerbated for streams in near-hand space. This is further evidence suggesting that attentional disengagement from stimuli is impaired in nearhand space (Abrams et al., 2008).

\section{Critical analysis of attentional and bimodal cell accounts}

Altogether then, the constellation of findings across visual search, IOR, and the AB led Abrams et al. (2008) to propose the detailed-evaluation account of altered visual processing near the hands, according to which visual processing is especially thorough and prolonged, thereby delaying attentional disengagement. Critically, the detailed-evaluation account predicts slower responses to visual stimuli near the hands. Thus, while this account draws on visual attention as a critical mechanism, like the attentional-prioritization account, these two accounts appear to use 'attention' to explain apparently contradictory patterns of results. That is, attentional prioritization predicts enhanced or facilitated responding to visual stimuli near the hands, not slowed visual search times, decreased IOR and exacerbated AB. Similarly, the detailed-evaluation account does not explain the facilitated responses found by Reed et al. (2006). Neither the attentional-prioritization (Reed et al., 2006, 2010) nor the detailed-evaluation account proposals (Abrams et al., 2008) compared or contrasted their theories with one another, and therefore neither offered an explanation for how these theories could account for the discrepant findings that led to the opposing theory.

One possible way in which the attentional prioritization and detailed-evaluation accounts could be reconciled is if they reflect two different stages of the same basic process. That is, visual stimuli in near-hand space may experience a baseline attentional prioritization, 
followed by thorough processing and delayed disengagement. However, such a hybrid account is weak theoretically, because it loses explanatory precision: it can be adapted posthoc to explain either a pattern of enhancement or facilitation. A theory which is sufficiently flexible to explain multiple different patterns of results is difficult to falsify, and therefore unsuitable as a scientific theory (Popper, 1959). As such, explanations of altered visual perception attributed to "attention” (e.g., Park, et al., 2013; Reed, et al., 2013) could be improved by greater theoretical precision. If attention is to satisfactorily explain patterns of processing in near-hand space, then these inconsistencies and imprecisions must first be addressed.

This same criticism applies to Reed et al.’s (2006, 2010) hypothesis that bimodal cells form the core neural correlate that can explain altered visual perception in near-hand space. On the one hand, the link between bimodal cells and near-hand space is obvious: bimodal cells have hand-centred receptive fields irrespective of hand visibility, which maps nicely onto the behavioural findings in near-hand space which are a function of proximity, and do not depend on hand visibility (Abrams et al., 2008; Weidler \& Abrams, 2013). Essentially, therefore, while offering a candidate explanation for why perception is altered in near-hand space, the bimodal cells account struggles to make more specific predictions about the nature of this alteration. On the other hand, although Reed et al. (2006, 2010) attempted to link these to attentional prioritization, this link was somewhat tenuous. And even if this link were upheld, bimodal cells are then unable to account for Abrams et al.’s (2008) results that appear to suggest especially detailed processing in near-hand space. Furthermore, Reed et al.'s (2013) recent evidence from event-related potentials (ERPs) found little evidence to support the notion of bimodal cells underlying generic faster responses to flashed targets. Finally, more recent evidence has come to light which further constrains the explanatory power of either attentional account or bimodal cells. 
One such piece of evidence that is difficult to explain with attention or bimodal cells is that from Davoli et al. (2010), who found a pattern of results consistent with the notion that semantic processing is impaired in near-hand space. In Experiment 1, participants' task was to determine whether visually-presented sentences were sensible (e.g., 'Tim carried his suitcase to the car') or nonsensical (e.g., 'Tim typed his suitcase to the car') and participants made their responses with their hands placed on response buttons next to the screen, or in their lap. Judgements of sensibleness were less accurate in near-hand space compared to farhand space. In Experiment 2, participants completed a Stroop task. Here their task is to report the colour of the text of words that are presented, and responses are less efficient (slower, and/or more error-prone) when the meaning of the word conflicts with the colour it is presented in (e.g., the word 'red' in appearing in blue) compared with when they do not conflict (e.g., the word 'red' appearing in red; Stroop, 1935). This classic Stroop interference for the incongruent versus congruent trials was attenuated in near-hand space (Davoli, et al., 2010). Contextualising their result in the previous literature, Davoli et al. (2010) suggested that near-hand space may experience a trade-off between spatial enhancement and semantic impairment. The semantic impairment in their results is evident. But it is difficult to simply summarise the previous literature as showing a spatial enhancement or facilitation, given the inconsistencies between attentional-prioritization (Reed et al., 2006, 2010) and detailedevaluation accounts (Abrams et al., 2008) and the respective empirical findings. However, a theory that was subsequently proposed is better able to account for the mix of 'spatial' findings, as well as elegantly incorporate the evidence for impoverished semantic processing in near-hand space. This theory drew upon the properties of the two major classes of visual cells, magnocellular neurons (M cells) and parvocellular neurons (P cells), whose properties are described below. According to this account, in near-hand space the input from $\mathrm{M}$ cells is enhanced, meaning that visual tasks that preferentially tap M-cell functions are facilitated, at 
the relative expense of $\mathrm{P}$ cell functions, and therefore tasks that rely on $\mathrm{P}$ cell functions are impaired.

\section{Magnocellular neuron (M-cell) Enhancement Theory}

$\mathrm{M}$ and $\mathrm{P}$ cells essentially represent a trade-off in temporal versus spatial acuity. Relative to P cells, M cells have faster conduction speeds, that is, they transmit information more quickly, and have greater temporal sensitivity, that is, sensitivity to rapid changes in luminance over time. This means that $\mathrm{M}$ cells are more sensitive to temporally-abrupt changes in stimuli, such as onsets, offsets, and motion, that is, sensitivity to rapid changes in luminance across time. $\mathrm{M}$ cells also have greater sensitivity to luminance contrast. In other words, $\mathrm{M}$ cells will fire in response to very low-contrast stimuli, whereas $\mathrm{P}$ cells require a higher contrast before they will fire. $\mathrm{M}$ cells have larger receptive fields, which allows them to process lower spatial frequencies (that generally corresponds to the "gist" of a scene or stimulus), resulting in lower spatial acuity. M cells are also virtually colour-blind. In contrast, P cells are more sensitive to rapid changes in luminance over space. P cells have smaller receptive fields, and they process higher spatial frequencies (that correspond to the finerresolution details of scene or stimulus), resulting in higher spatial acuity. P cells are responsible for virtually all colour processing. In a nutshell, $\mathrm{M}$ cells have high temporal acuity but poor spatial acuity, whereas $\mathrm{P}$ cells have high spatial acuity but poor temporal acuity (Denison, Vu, Yacoub, Feinberg, \& Silver, 2014; Derrington \& Lennie, 1984; Legge, 1978; Livingstone \& Hubel, 1988). In real-world vision, both M and P cells collaborate to create and update our dynamic conscious perception of objects and scenes. However, given stimulus or task requirements, the relative balance of the contribution of $\mathrm{M}$ versus $\mathrm{P}$ cells may change. 
$\mathrm{M}$ and $\mathrm{P}$ cells are arranged in channels, which form a pathway from the retina to the striate cortex of the brain, via the lateral geniculate nucleus (LGN) in the thalamus. From the striate cortex, these channels form predominate although not exclusive connections to the dorsal and ventral cortical processing streams respectively (Livingstone \& Hubel, 1988; Merigan \& Maunsell, 1993; Schiller \& Logothetis, 1990). The dorsal stream projects into posterior parietal regions, terminating at the motor cortex, whereas the ventral stream reaches into the inferotemporal (IT) cortex in the temporal lobe, which includes object-selective cortical regions (Gauthier, Tarr, Anderson, Skudlarski, \& Gore, 1999; Grill-Spector, Kourtzi, \& Kanwisher, 2001; Grill-Spector, Tammar, Hendler, \& Malach, 2000; Kanwisher \& Yovel, 2006). The dorsal cortical stream is implicated in the visual control of motor behaviour (e.g., reaching), and the shifting of attention in space, and motion-processing, whereas the ventral stream is implicated in the identification and recognition of objects (Goodale \& Milner, 1992; Goodale \& Westwood, 2004; Mishkin \& Ungerleider, 1982; Mishkin, Ungerleider, \& Macko, 1983; Vidyasagar, 1999). The majority of $M$ cells are found in the dorsal stream, whereas most P cells are found in the ventral stream (Maunsell, Nealey, \& DePriest, 1990; Merigan \& Maunsell, 1993; Nassi \& Callaway, 2007).

Gozli et al. (2012) proposed that near-hand space was the purview of enhanced M-cell input (see also Previc, 1990). This account predicts a spatiotemporal trade-off in precision in near-hand space: impaired performance when the task requires spatial acuity and enhanced performance when the task requires temporal precision. To test this notion, Gozli et al., (2012) had participants perform a spatial and temporal gap discrimination task in near-hand (respond via mice attached to the side of the computer monitor) versus far-hand space (respond via keyboard on the desk beneath the computer). Specifically, the spatial-gap detection task required participants to judge whether a briefly-presented stimulus was an intact circle, or had a small spatial gap in it. This requires spatial acuity, and therefore 
preferential $\mathrm{P}$ cell function. The temporal-gap detection task required participants to determine whether a circle was presented continuously across time, or whether there was a brief blank interval in between two presentations of the circle. This task requires temporal acuity, and therefore preferentially taps $\mathrm{M}$ cell function. Such tasks have been successfully used previously to differentiate M and P cell function (e.g., Bocanegra \& Zeelenberg, 2011).

As predicted by the M-cell enhancement account, Gozli et al. (2012) observed impaired spatial acuity but improved temporal acuity in near-hand space relative to far-hand space. Moreover, there was no overall difference in accuracy or RT between near and farhand space, instead only this trade-off of between spatial and temporal acuity between the hand proximity conditions was evident (Gozli et al., 2012). Neither attentional prioritization (Reed et al., 2006, 2010), nor detailed evaluation (Abrams et al., 2008) can explain this pattern of results.

Gozli et al. (2012) pointed out that the M-cell enhancement account could also potentially explain the pattern of results observed in previous studies. Reed et al.'s (2006, 2010) finding of enhanced RTs to targets could have reflected the fact that since the task in these studies was merely to detect the onset of a square (coarse spatial acuity), and the onset of these targets was signalled by a rapid change in luminance, M-cells would convey this information more efficiently. The M-cell enhancement account could, therefore, offer a posthoc explanation for the RT facilitation observed in near-hand space. Of course, these target stimuli were presented at $100 \%$ contrast, and thus $\mathrm{P}$ cells would also be capable of processing them. M-cells, however, with their more rapid conduction speed, would process this information more quickly, and therefore this could potentially facilitate target detection RTs. Note that a major difference between this and Gozli et al. (2012) described above was that in Reed et al.'s study responses were speeded, and thus this could be why M-cell enhancement manifested as an RT advantage here, whereas it was an accuracy difference in the absence of 
an RT difference in Gozli et al. (2012). In a similar vein, Abrams et al.’s (2008) visual search and AB tasks both used alphanumeric stimuli, which Gozli et al. (2012) suggest constitute high spatial frequencies (but see critique in section synthesising attentional and neurophysiological explanations). But if this is so, then since $\mathrm{M}$ cells are not well suited to processing high spatial frequencies, this could therefore explain the poorer performance (e.g., increased $\mathrm{AB}$ ) in near-hand space. There are also many other subsequent findings that dovetail nicely with the M-cell account, and are difficult to reconcile with either attentional account, even if this has been offered as the theoretical motivation or explanation. These cases are discussed fully below.

It has been shown that near-hand space affects the segregation of objects from backgrounds. Specifically, Cosman and Vecera (2010) demonstrated that regions that were reached towards were more likely than other regions to be assigned as foreground figures. At the time, this was attributed to "attention". However, $\mathrm{M}$ cells facilitate figure-ground segmentation (Livingstone \& Hubel, 1988), and therefore this finding can be incorporated within the M-cell enhancement framework.

Furthermore, Goodhew et al. (2013a) used an object-substitution masking paradigm, in which participants' perception of a briefly-presented target surrounded by four-dots is impaired ('masked') when the four-dots remain visible after the target disappears (Enns \& Di Lollo, 1997; Di Lollo, Enns, \& Rensink, 2000). Masking reflects the failure of visual system to segment the target and trailing mask as distinct objects across time (for a review, see Goodhew, Pratt, Dux, \& Ferber, 2013b). Goodhew et al. (2013a) found that masking was reduced when the visual stimuli were in near-hand space. These authors were agnostic about whether the attention-based or M-cell enhancement could explain the reduced masking. However, evidence that has come to light more recently places the finding of reduced objectsubstitution masking in near-hand space squarely in favour of the M-cell enhancement 
account. That is, while there was initially some debate about this issue, the evidence appears to suggest that object-substitution masking magnitude is not modulated by attention (Argyropoulos, Gellatly, Pilling, \& Carter, 2013; Filmer, Mattingley, \& Dux, 2014; Pilling, Gellatly, Argyropoulos, \& Skarratt, 2014), whereas it is directly modulated by M-cell involvement, such that masking is increased when M cells are saturated (Goodhew, Boal, \& Edwards, 2014). Thus, the fact that object substitution masking magnitude is increased when M cells are suppressed, and is unaffected by attentional manipulations, implies that the reduction of masking in near-hand space (Goodhew et al., 2013a) supports the notion of enhanced M-cell input in near-hand space.

As discussed above, $\mathrm{M}$ and $\mathrm{P}$ cells have differential spatial-frequency sensitivities. Abrams and Weidler (2013) found that participants were less sensitive to briefly-presented high spatial-frequency Gabors, whose orientation they were discriminating, in near-hand space relative to far-hand space, but more sensitive to the low spatial-frequency Gabors in near-hand space. Moreover, this difference was eliminated by the application of red diffuse light, which inhibits $\mathrm{M}$ cells, because of the presence of a subset of on-centre off-surround $\mathrm{M}$ cells whose surround is inhibited by red light across the receptive field (de Monasterio, 1978; Dreher, Fukada, \& Rodieck, 1976; Wiesel \& Hubel, 1966). Similarly, it has been shown that the classification of line drawings of objects (i.e., judging whether the object is bigger or smaller than a typical shoebox) is facilitated when the images are achromatic and lowcontrast (i.e., M-cell preferred) relative to when are chromatically defined and isoluminant (Kveraga, Boshyan, \& Bar, 2007). Chan et al. (2013) found that this difference in processing time between $\mathrm{M}$ and $\mathrm{P}$ cell preferred images was exacerbated in near-hand space. Thus, all of these results can be encapsulated by the theory of M-cell enhancement. Neither the attentional prioritization (Reed et al., 2006, 2010) nor the detailed-evaluation (Abrams et al., 2008) accounts can explain this pattern of results, as they predict uniform 
enhancement/impairment across all tasks, rather than differential performance depending on spatial frequency content. Also, since attention is unrelated to red diffuse light, they offer no explanation for its effect on visual perception in near-hand space.

Furthermore, as mentioned previously, P cells subserve virtually all of the processing of colour information, whereas $\mathrm{M}$ cells, while some are inhibited by red light, are almost colour-blind. Further evidence for M-cell enhancement in near-hand space, therefore, is that colour perception is adversely impacted for visual stimuli in near-hand space (Goodhew, Fogel et al., 2014; Kelly \& Brockmole, 2014). Specifically, Goodhew, Fogel et al. (2014) used a change-detection task which gauges the independent contribution of colour and spatiotemporal-trajectory to object-correspondence across temporary occlusion (Hollingworth \& Franconeri, 2009). There was a transfer effect, whereby colour did not affect reaction time to correctly identify the absence of a change for those participants who completed the handsnear condition first, and this absence of colour processing carried over to subsequent handsfar visual processing. In contrast, colour affected responses in both near and far-hand space for those participants who completed the hands-far condition first. This suggests that nearhand space impairs colour processing, and at least when the same response-apparatus is used in both conditions, there is the potential for this pattern to transfer across time. There were no overall differences in change-detection accuracy or response time between near-hand and far-hand space, therefore contradicting an attention-based explanation. Similarly, Kelly and Brockmole (2014) used a change-detection task, and found that participants' ability to report changes in colour information was impoverished in near-hand space, whereas their ability to report orientation was increased. This pattern of processing in near-hand space is consistent with Gozli et al.'s (2012) M-cell enhancement account of altered visual processing near the hands. In contrast, neither of the attention-based accounts could explain this, as they predict a 
uniform enhancement or decrement of visual processing, rather than a trade-off dependent on whether the stimulus property in question is $\mathrm{M}$ or P-cell preferred.

Along the same lines, Gozli et al. (2014) found evidence for reduced feature-binding in near-hand space across two experiments. In their first experiment, these authors found that the object-specific preview effect, which reflects the continuation of an object identity across spatiotemporal interruption (Kahneman, Treisman, \& Gibbs, 1992), was attenuated in nearhand relative to far-hand space. In their second experiment, there was a shape-based priming effect specific to near-hand space. These two findings are difficult to reconcile with attentional accounts. But they can be explained by the M-cell enhancement account, if, as Gozli et al. (2014) suggest, the dorsal cortical stream just requires individual features rather than bound objects in order to guide action (Barense, Gaffan, \& Graham, 2007; Ganel \& Goodale, 2003). Given the strong connections between M cells and the dorsal cortical stream, reduced feature binding is consistent with the M-cell enhancement account of altered visual perception near the hands.

At first blush, the M-cell enhancement account may appear counter-intuitive. Why would the visual system be tuned so as to impair the processing of fine details near the hand? This would make the visual control of fine motor tasks in the hands, such as threading a needle, inordinately difficult. One possibility is that because objects near the hands are candidates for action, their location, motion, and coarse outline are more important properties to process than their finer spatial details. However, another possibility is that rather than being directly selected-for, these effects may be a by-product of other selected-for properties, such as the fact that the near-hand space, by definition, implicates integrating dynamic information about the location of the hands in space (body-centred) with visual information about the external world. Since the motor cortex sits at the termination of the dorsal stream, 
and $\mathrm{M}$ cells represent the majority input into the dorsal stream, the connection between enhanced $\mathrm{M}$ cell input and perception in near-hand space may simply be a vestige of this link.

Altogether then, there is a growing body of evidence that is consistent with the M-cell enhancement account of altered perception in near-hand space. In contrast, the attentional accounts and bimodal cell account appear to contradict one another, and either in isolation or even in conjunction cannot explain the pattern of results emerging in this field. This is not to say that bimodal cells are not implicated in some way in near-hand space effects - their ability to encode combined visual and tactile and information may help the system to dynamically update the location of the hands in space in order to keep as accurate registration of what region constitutes near-hand space. This would then allow the system to dampen the signal from $\mathrm{P}$ cells and possibly the ventral stream, and upregulate the signal from $\mathrm{M}$ cells and possibly the dorsal stream in near-hand space. However, bimodal cells are unable to account for patterns of performance, since they may only serve a role of mediating the effect of other types of cells. Most importantly, it is not valid to equate the attentional and bimodal cell accounts on the one hand with the M-cell based account on the other, as they make quite distinct predictions about patterns of visual processing and performance. However, despite the growing evidence for $\mathrm{M}$-cell enhancement and evidence that the attentional accounts cannot explain, there remain some results that do not as clearly fit within this theoretical framework, which are discussed below.

\section{Results for further theoretical analysis}

As noted above, the M-cell enhancement account offers an alternative possible explanation (to detailed-evaluation theory) for Abrams et al.'s (2008) slowed visual search and poorer $\mathrm{T} 2$ identification in the $\mathrm{AB}$, if we assume that these paradigms used high spatial frequency alphanumeric stimuli, unsuitable for M-cell processing (but see section on 
synthesising attentional and neurophysiological explanations for a critique of this assumption). The finding of reduced IOR in response to flashed cues, however, is more difficult to conceptualise as M-cell enhancement. That said, given the weight of evidence emerging in support of M-cell enhancement, what this finding might tell us is that P-cells facilitate attentional disengagement from stimuli, and thus the reduced IOR was a product of a relative deficit of P-cell input in near-hand space. Alternatively, it may be that a synthesis of attentional mechanisms and neurophysiological mechanisms is required in order to explain the full gamut of behavioural results observed in near-hand space. In the next section (“synthesising attentional and neurophysiological explanations”) we offer some suggestions for how this synthesis might occur. A complete understanding of the mechanism(s) underlying reduced IOR in near-hand space might await, or perhaps even inform, this development.

Another such study that requires further empirical testing and theoretical analysis is that of Tseng and Bridgeman (2011), who tested participant's visual short-term memory (VSTM) in near and far-hand space. This involved a standard change-detection task, in which participants' task is to detect whether there has been a change between presentations of a given array. On half of trials there is a change, which consists of one the squares changing colour. This task measures VSTM (Luck \& Vogel, 1997). Tseng and Bridgeman (2011) found that change detection sensitivity was improved in near-hand space. These authors interpreted this result as reflecting with a "deeper focus of attention of objects near the hands, which allows one to detect rapid onset faster and encode them into visual working memory deeper, but at the cost of longer attentional shift when distracters are present due to the equally extensive visual analysis of all items” (p. 268), i.e., consistent with the detailedevaluation account. 
Of course, when it comes to higher cognitive processes like visual memory, a simple $\mathrm{M}$ versus $\mathrm{P}$ dichotomy breaks down and more complex cortical mechanisms that draw on multiple cell types come into play. That said, however, a 'front-end' deficit in visual processing (e.g., an inability to discriminate between different colours when colour is the property that defines the different stimuli in the array) would have a direct impact on task performance, independent of any memory effects. In this vein, the finding of enhanced colour change-detection in near-hand space could be said to be at odds with an M-cell enhancement account, which might predict the opposite since M-cells do not process colour. However, this theory can be reconciled with this finding by noting that the colours used in the display were not matched for luminance, and indeed, black and white were used as possible colour options in the VSTM array (Tseng \& Bridgeman, 2011). This means that even though it was designed as a colour change-detection, it could have become a luminance change detection task. Given that $\mathrm{M}$ cells are sensitive to changes in luminance across time, but not colour, then $\mathrm{M}$ cells could have subserved this processing. It is interesting to note that in the studies that did find impaired colour perception in near-hand space (Goodhew, Fogel et al., 2014; Kelly \& Brockmole, 2014), black and white were not possible stimulus colours, meaning that the stimuli would have been more similar in luminance than those in Tseng and Bridgeman's (2011) experiments. That said, while M-cells have lower contrast detection thresholds, at full contrast changes (e.g., black to white), both $\mathrm{M}$ and $\mathrm{P}$ cells would both be capable of processing such changes, and therefore the link between $\mathrm{M}$ and $\mathrm{P}$ cell processing and performance on this visual memory task may be somewhat tenuous. Future research, therefore, is required in order to disentangle the front-end contributions of $\mathrm{M}$ and $\mathrm{P}$ cells to basic processing of the stimuli, versus how higher-level cognitive processes like visual memory might be impacted by hand proximity. 
Moreover, Adam et al. (2012) had participants identify a briefly-presented and masked letter, while they were moving their hands in a cyclical bimanual movement, which manipulated the proximity of the hands to the visual stimuli in a spatially-graded rather than dichotomous manner. Adam et al. reported that letter identification was improved when the hands were near the visual stimuli relative to when they were in an intermediate position, and further impaired when their hands were far (i.e., a spatially-graded facilitation effect). Given that the target stimuli here were small letters (i.e., high spatial-frequency stimuli), this facilitation effect would appear to contradict the M-cell enhancement account, and could be indicative of support for attentional prioritization. However, there are two possible explanations for this anomaly that fit within the M-cell enhancement framework.

Firstly, it has been established that M-cells facilitate segmenting the target and mask as distinct objects when they are presented in close spatiotemporal proximity, thereby protecting the target from masking (Goodhew, Boal, et al., 2014). In other words, visual masking can be conceptualised as an erroneous inference of object integration in the face of dynamic input. That is, when bombarded with ongoing dynamic input, the visual system needs to nonconsciously infer what information belongs to a single object continuing over time (object integration), versus what information belongs to two or more distinct objects despite their close spatial and temporal proximity (object segmentation). When the target fails to be perceived in visual masking, the evidence suggests that it can reflect the integration of the target with the after-coming mask, such that the mask predominates in conscious perception at the expense of the target (Coltheart \& Arthur, 1972; Goodhew, Pratt, et al., 2013). The greater the tendency for the visual system to segment, that is, treat the target and mask as independent objects, the weaker (or even absent) masking will be (e.g., Goodhew, Edwards, Boal, \& Bell, 2015; Lleras \& Moore, 2003; Luiga \& Bachmann, 2008; Moore \& Lleras, 2005). M-cells, with their superior temporal resolution, facilitate object segmentation, thereby 
weakening masking (Goodhew, Boal, et al., 2014). Therefore, given that in Adam et al.'s (2012) experiment the letters were presented briefly and masked, M-cell enhancement in near-hand space may have helped reduce masking, thereby improving letter identification.

Secondly, Festman and colleagues (Festman, Adam, Pratt, \& Fischer, 2013a, 2013b) suggested that the apparent facilitation effect observed in Adam et al. (2012) was a product of averaging over different movement trajectories (i.e., moving toward and away from the visual stimuli), and found that when these are disentangled, letter discrimination was most strongly increased when the hand were far from the visual stimulus location, but moving toward it. The fact that letter identification here was improved in far-hand space is consistent with the M-cell enhancement account. These stimuli, however, were not designed to be optimal for selectively gauging $\mathrm{M}$ versus $\mathrm{P}$ cell function, as this was not their intended purpose, and therefore future research is required to support this interpretation. Moreover, if this explanation were supported, then the M-cell enhancement theory would need to be extended, in order to incorporate the effect of movement trajectory.

In addition to the evidence from healthy participants, evidence from neuropsychological cases has the potential to inform our understanding of the mechanisms underlying altered visual perception in near-hand space. Schendel and Robertson (2004) tested patient WM, who suffers cortical "blindness" as a result of damage to his right primary visual cortex, in order to test the effect of hand proximity on visual perception in his impaired visual field. These authors found that WM's ability to detect the onset of an unfilled blue circle target was improved when his arm was extended towards the screen compared to when his hand was placed in his lap. Given that the onset of the target reflects a rapid change in luminance, and the target was not full contrast, this could be interpreted as consistent with either attentional prioritization or M-cell enhancement in near-hand space. Further testing of WM would be able to delineate these possibilities. For example, if WM's performance is improved on 
luminance detection and flicker detection but not static HSF stimulus detection, then this would be clearer evidence in favour of the M-cell enhancement account. In contrast, if WM's perception is improved both in terms of flicker detection and static HSF stimulus detection acuity, then this would be evidence for attentional prioritization.

\section{Synthesising attentional and neurophysiological explanations}

The motivation for this review arose from some confusion and ambiguity in the literature about equating existing attentional and M-cell enhancement accounts, despite the fact that they make contrasting predictions about performance for different types of visual tasks. For example, the M-cell enhancement account correctly predicts the impairment on spatial gap discrimination tasks for near relative to far-hand space, whereas attentional prioritization would predict improved perceptual performance here. It is not valid, therefore, to merely equate $\mathrm{M}$ cells and attention with no further explanation. However, there is one possible way in which they could meaningfully be linked: attentional spotlight size. M cells have much larger receptive fields than P cells, and therefore near-hand space may implicate a more "diffuse" attentional spotlight over a broader area than far-hand space. If this is so, then not only might this help extend our knowledge of visual perception near the hands, but it could also inform the mechanisms of visual attention more broadly.

That is, in natural scenes, the amount of available visual information far exceeds the limited cognitive processing capacity of the brain. This means that we can be consciously aware of only a subset of objects in the visual scene. For example, when walking down a crowded street, there are too many faces, cars, signs, and shopwindows to process simultaneously. In these circumstances, spatial attention serves as a filter, selecting some objects for enhanced processing, which can ultimately result in conscious perception of those objects. For instance, we may attend to and read just one sign from the busy street at a given 
point in time. Until now, the prevailing metaphor for conceptualising the function of spatial attention has been that of a spotlight: a small, focussed area of enhanced processing (Eriksen \& Hoffman, 1972; Grubert, Righi, \& Eimer, 2013; Tsal \& Lavie, 1988). Decades of research have demonstrated that the location of this spotlight can be shifted in space to select features, objects, or locations (Carrasco, 2011; Folk, et al., 1992; O'Craven, Downing, \& Kanwisher, 1999; Posner, 1980; Posner \& Cohen, 1984; Wyble, et al., 2013), and that this shifting can be dissociated from eye movements (“covert attention”). This is important because the functional enhancement provided by the spotlight is especially crucial when objects are not at the point of fixation. In this way, it has at times been implicitly assumed that spatial attention confers a universal enhancement to the processing all aspects of the attended object. This implicit notion of universal enhancement, however, is refuted by evidence which shows that cueing covert attention to location impairs temporal acuity and low spatial-frequency processing at that location (Enns, Brehaut, \& Shore, 1999; Yeshurun \& Carrasco, 1998; Yeshurun \& Hein, 2011; Yeshurun \& Levy, 2003; Yeshurun \& Marom, 2008). This, in conjunction with a plethora of evidence that cueing attention to a location improves spatial acuity (for a review see Carrasco, 2011), indicates that a covert shift of attention precipitates a bias toward parvocellular processing (Yeshurun, 2004; Yeshurun \& Rashal, 2010; Yeshurun \& Sabo, 2012). Although modifying the size of the attentional spotlight reflects a different mechanism to that of covert shifts of the spotlight, this biological basis for understanding the consequence of a covert shift may shed some light on the likely mechanism behind instantiating different spotlight sizes.

It has been established that it is possible to modify the size of the attentional spotlight, which has both behavioural and neurophysiological consequences (Greenwood \& Parasuraman, 1999, 2004; LaBerge, 1983; Muller, Bartelt, Donner, Villringer, \& Brandt, 2003). At least when it comes to spatial acuity, it appears that increasing the size of the 
attentional spotlight decreases the resolution of encoding of items within that spotlight, akin to zoom-lens trade-off between area and precision (Eriksen \& St. James, 1986; Eriksen \& Yeh, 1985). To date this has been tested exclusively with high spatial-frequency content tasks, such as letter discrimination. It remains to be seen, therefore, whether there is a spatiotemporal trade-off as spotlight size increases. That is, M and P cells, with their different receptive field sizes, may be the way in which the visual system instantiates different spotlight sizes. In other words, in order to instantiate a small, "focal" attentional spotlight, P cells (with their smaller receptive fields) could be preferentially called upon, which would lead to enhanced spatial and impaired temporal acuity. In contrast, in order to instantiate a large, “diffuse” spotlight, input from $\mathrm{M}$ cells (with their larger receptive fields) could be selectively upregulated. If so, then this would mean that under a diffuse attentional spotlight, temporal resolution would be enhanced at the cost of spatial acuity. This would implicate a qualitative trade-off between spatial and temporal acuity as function of attentional spotlight size, which would fundamentally challenge the zoom-lens notion that a more focussed spotlight universally benefits all aspects of visual processing, and that diffusing the spotlight merely dilutes this benefit.

In fact, recent evidence at least indirectly suggests that this might be the case. Specifically, Bush and Vecera (2014) found that the trade-off between spatial and temporal in near-hand space first reported by Gozli et al. (2012), qualitatively shifts as a function of whether two or one hands are placed next to the display. When two hands were next to the display, these authors replicated the advantage for temporal and disadvantage for spatial acuity (relative to no hands near the display). When just one hand was placed next to the display, and "near-hand space" was defined as the side of the screen adjacent to the hand, then spatial acuity was enhanced and temporal acuity impaired (Bush \& Vecera, 2014). These authors suggested that the hands might provide the 'frame' that determines the size of the 
attentional spotlight. That is, the single-hand set-up induces a small, focal spotlight, instantiated via $\mathrm{P}$ cells, and the dual-hand set-up induces a large, diffuse spotlight, instantiated via M-cells. This, therefore, explains the trade-off between spatial and temporal acuity as hand set-up changes. Why would the system shift the balance based on one versus two hands near an object? We suggest a novel possibility: that two-handed interactions with objects tend to require coarser spatial information (e.g., carrying a large object, or deflecting a swooping bird), whereas single-handed interaction with objects might require finer spatial resolution (e.g., picking a particular berry from amongst others on a bush, or pulling a splinter out from under the skin). These different functional requirements for different handobject relations may be the reason why the system adaptively shifts between magnocellular and parvocellular biased processing.

Given that the processing bias in near-hand space appears to flexibly adapt toward a parvocellular or magnocellular bias dependent on the relationship between the hand(s) and the object, could other aspects, such as the visual task that one is performing, irrespective of the one versus two hand manipulation, affect this relative balance? We have reason to believe that the attentional demands of the task can determine the relative balance of $\mathrm{M}$ versus $\mathrm{P}$ cell processing. A key piece of evidence comes from a reconsideration of Abrams et al.'s (2008) finding of slowed visual search in near-hand space. Recall that Gozli et al. (2012) retrospectively classified the stimuli used in this task is HSF, because they were letters. However, whether a given stimulus is high or low spatial frequency depends critically on its size. Closer examination reveals that Abrams et al.'s stimuli were large and clearly LSF: they subtended $3^{\circ} \times 1.5^{\circ}$ of visual angle. Stimuli of an equivalent total size (e.g., $1.93^{\circ} \times 2.34^{\circ}$ ) have been used to create a 'global' (vs local) stimulus in compound Navon figures (Hubner, 1997), the processing of which is mediated via LSFs (Shulman, Sullivan, Gish, \& Sakoda, 1986). Given, then, that the stimuli used in the visual search task at least contained (if not 
predominately reflected) LSFs and therefore effectively drove M-cells, the fact that visual search was found to be less efficient in near-hand space is actually consistent with a parvocellular processing bias in near-hand space.

What is it about the visual search task that would induce a shift toward parvocellular processing? We suspect it has to do with attentional spotlight size adapting to the cluttered arrays and the demands of the search task. With a large visual search array there are a large number of objects, and the potential for crowding and perceptual confusion is high. Imagine a diffuse attentional spotlight applied to such an array: many different objects might be contained within its bounds, creating interference and potential confusion about object identity. A small spotlight, in contrast, would be optimal, as it would minimise of interference from multiple objects simultaneously within the attentional spotlight, allowing the identity of the object attended at a given point in time to be effectively resolved. This small spotlight could then be moved around to consider each of the objects in the array until the target is found.

Given this evidence for a parvocellular bias, how do we reconcile this with the existing evidence? The likely reason why a clear M-cell bias has been obtained in other experiments is that the typical stimuli and tasks have been stripped down and simplistic, such as the requirement to identify a single Gabor or ring presented alone in the centre of the screen (e.g., Abrams \& Weidler, 2013; Gozli et al., 2012). Where there have been multiple items in the display, such as distractors in object-substitution masking (Goodhew, Gozli, et al., 2013), the target was very clearly signalled with a salient pop-out cue (the only object in the array with four-dots around it), negating any real visual search requirement. In visual search, in contrast, there is likely a benefit to constricting the size of the attentional spotlight and thereby probably shifting to a parvocellular bias. In further support of the notion that visual search demands can determine the balance of cellular processing, we recently found 
that visual search for targets in arrays of high-spatial frequency Gabors was facilitated in near-hand space, but only when the set-size was large (Goodhew \& Clarke, revision under review). To summarise then, the most comprehensive framework for understanding the patterns of results observed is that under low attentional demands, there is a magnocellularbias in near-hand space, whereas under high attentionally-demanding conditions, there is a parvocellular bias in near-hand space.

This framework offers exciting new opportunities for future research. What constitutes "high attentional demands"? Here we offered a preliminary definition based on the need for selective attention to identify a target amongst multiple distractors. With Bush and Vecera (2014) we saw that that having just a single hand near the stimulus, possibly because this signals the need for fine spatial resolution in order to interact with the object. There are very likely other factors, however, that can also affect the relative balance of $\mathrm{M}$ versus P-cell processing, and it would be useful for future research to uncover what these factors are. It seems likely, however, that attention, and spotlight size in particular, may play a critical role. Ultimately, therefore, the mechanism underlying altered visual perception near the hands is likely to be a combination of attention and biases toward magnocellular versus parvocellular processing.

In this vein, it would also be useful to reconsider Abrams et al.’s (2008) finding of an exacerbated attentional blink in near-hand space. At this point it is unclear whether this task was M-cell or P-cell preferred, as the alphanumeric characters used were of intermediate size $\left(1.6^{\circ} \times 1.75^{\circ}\right)$. Future research could compare performance in the AB for stimuli tailored to be LSF and HSF for near versus far-hand space. Since the AB measures the allocation of attentional resources across time, rather than space (e.g., Dux \& Marois, 2009), understanding the effect of hand proximity on $\mathrm{AB}$ performance would elucidate how $\mathrm{M}$ versus P cells contribute to the allocation of attentional resources across time. 
Another way in which attention-based explanations could gain theoretical traction is to focus on existing neurophysiological explanations of the effect of attention. That is, the putative changes in spatial and temporal acuity as spotlight size changes described above, represents a biologically-based model of attention. There are other biologically-based models of attention, however, which have already been proposed in the literature independent of the near-hand space literature. For example, in an attempt to reconcile conflicting effects of emotional stimuli on performance in attentional tasks, Bocanegra (2014) proposed that there is a trade-off between M-cell and P-cell mediated perception on the one hand and speed versus accuracy on the other. That is, this model stipulates that responses on visual attentional tasks will be faster to M-cell-mediated coarse-grained spatial perception, whereas responses will be more accurate to P-cell-mediated fine-grained spatial perception. This model does not have great explanatory power for the performance effects observed in near-hand space, because accuracy, rather than response time, is affected for both spatial frequencies improved accuracy for LSF in near-hand space (with a two-hand set-up) and impaired accuracy for HSF in near-hand space (Abrams \& Weidler, 2013). The same is true for spatial versus temporal acuity - accuracy, rather than response time, is impacted for both, although in different directions (Gozli et al., 2012). The model does highlight, however, the emerging consensus in a number of domains that models espousing generic effects of attention are limited in their explanatory power, whereas neural-based models that implicate trade-offs between different aspects of processing appear to have the strongest theoretical merit. For near-hand space, however, the clearest trade-off is between M-cell and P-cell mediated perception, predominately on measures of accuracy. As discussed above, however, attentional demands appear to determine the balance point between $\mathrm{M}$-cell and P-cell mediated perception. 
Another valuable avenue for future research is scale up from a focus on parvocellular versus magnocellular mediated perceptual tasks, to those that tap ventral and dorsal cortical processes. As noted earlier, $\mathrm{M}$ cells represent the majority input into the dorsal stream and $\mathrm{P}$ cells the majority input into the ventral stream, but this mapping is not exclusive (e.g., Nassi \& Callaway, 2007). Dorsal-mediated functions (e.g., object orientation processing, localisation of object in space and time) and ventral-mediated tasks (e.g., object recognition) are those that more closely parallel the sorts of real-world tasks that people perform, to a greater extent than tasks such as pure spatial versus temporal gap detection. Understanding how performance on these tasks fares and varies as a function of attentional load and task demands will offer useful insights into how altered perception near the hands impacts on our everyday life.

\section{Conclusion}

As reviewed above, there has been a pervasive lack of predictive precision when 'attention' is invoked as an explanation for altered visual processing near the hands. Attentional prioritization (Reed et al., 2006; 2010) and detailed evaluation and prolonged disengagement (Abrams et al., 2008) appear to contradict one another, and both have difficulty explaining many of the emerging findings, which implicate a trade-off whereby spatial acuity and colour perception is impaired while temporal resolution is enhanced in near-hand space, consistent with the M-cell enhancement account (Gozli et al., 2012). While there is growing evidence for the M-cell account, both critical re-evaluation of existing evidence and findings from recent studies converge to indicate that the demands of the task at hand, and whether one or two hands are near the object, may compel a small or large attentional spotlight, which, in turn, selectively upregulates the contribution of $\mathrm{M}$ versus Pcells. For example, under attentionally-demanding visual search conditions, a small spotlight is advantageous, and this then recruits a bias toward parvocellular processing. The evidence 
suggests that this is the case. Ultimately, therefore, it appears that a synthesis of attention and the underlying neurophysiology offers the most comprehensive explanation for altered visual perception near the hands. 


\section{Acknowledgements}

This research was supported by an Australian Research Council (ARC) Discovery Early Career Research Award (DE140101734) awarded to S.C.G., ARC Discovery Project (DP110104553) awarded to M.E, a Natural Sciences and Engineering Research Council of Canada (NSERC) Discovery Grants awarded S.F. and J.P. Correspondence regarding this study should be addressed to Stephanie Goodhew (stephanie.goodhew@anu.edu.au), Research School of Psychology, The Australian National University. 


\section{References}

Abrams, R. A., Davoli, C., Du, F., Knapp, W. H., \& Paull, D. (2008). Altered vision near the hands. Cognition, 107(3), 1035-1047. doi: 10.1016/j.cognition.2007.09.006

Abrams, R. A., Davoli, C. C., Du, F., Knapp, W. H., III, \& Paull, D. (2008). Altered vision near the hands. Cognition, 107(3), 1035-1047. doi: 10.1016/j.cognition.2007.09.006

Abrams, R. A., \& Weidler, B. J. (2013). Trade-offs in visual processing for stimuli near the hands. Attention, Perception, \& Psychophysics, 76(4), 1242-1252. doi:

$10.3758 / \mathrm{s} 13414-013-0583-1$

Adam, J. J., Bovend'Eerdt, T. J., van Dooren, F. E., Fischer, M. H., \& Pratt, J. (2012). The closer the better: Hand proximity dynamically affects letter recognition accuracy. Attention, Perception, \& Psychophysics, 74(7), 1533-1538. doi: 10.3758/s13414-0120339-3

Argyropoulos, I., Gellatly, A., Pilling, M., \& Carter, W. (2013). Set size and mask duration do not interact in object-substitution masking. Journal of Experimental Psychology: Human Perception and Performance, 39(3), 646-661. doi: 10.1037/a0030240

Barense, M. D., Gaffan, D., \& Graham, K. S. (2007). The human medial temporal lobe processes online representations of complex objects. Neuropsychologia, 45(13), 29632974. doi: 10.1016/j.neuropsychologia.2007.05.023

Becker, S. I., Folk, C. L., \& Remington, R. W. (2010). The role of relational information in contingent capture. Journal of Experimental Psychology: Human Perception and Performance, 36(6), 1460-1476.

Bocanegra, B. R. (2014). Affecting speed and accuracy in perception. Cognitive, Affective \& Behavioral Neuroscience, 14(4), 1454-1466. doi: 10.3758/s13415-014-0296-5. 
Bocanegra, B. R., \& Zeelenberg, R. (2011). Emotion-induced trade-offs in spatiotemporal vision. Journal of Experimental Psychology: General, 140(2), 272-282. doi: $10.1037 / \mathrm{a} 0023188$

Broadbent, D. E. (1958). Perception and communication. Elmsford, New York: Pergamon Press.

Brockmole, J. R., Davoli, C., Abrams, R. A., \& Witt, J. K. (2013). The world within reach: Effects of hand posture and tool use on visual cognition. Current Directions in Psychological Science, 22(1), 38-44. doi: 10.1177/0963721412465065

Brozzoli, C., Gentile, G., \& Ehrsson, H. (2012). That's near my hand! Parietal and premotor coding of hand-centered space contributes to localization and self-attribution of the hand. The Journal of Neuroscience, 32(42), 14573-14582. doi:

10.1523/JNEUROSCI.2660-12.2012

Bush, W. S., \& Vecera, S. P. (2014). Differential effect of one versus two hands on visual processing. Cognition, 133(1), 232-237. doi: 10.1016/j.cognition.2014.06.014

Carrasco, M. (2011). Visual attention: The past 25 years. Vision Research, 51(13), 14841525. doi: 10.1016/j.visres.2011.04.012

Chan, D., Peterson, M. A., Barense, M. D., \& Pratt, J. (2013). How action influences object perception. Frontiers in Psychology, 4, 462. doi: 10.3389/fpsyg.2013.00462

Coltheart, M., \& Arthur, B. (1972). Evidence for an integration theory of visual masking. Quarterly Journal of Experimental Psychology, 24(3), 262-269. doi: $10.1080 / 14640747208400279$

Cosman, J. D., \& Vecera, S. P. (2010). Attention affects visual perceptual processing near the hand. Psychological Science, 21, 1254-1258. 
Davoli, C. C., Du, F., Montana, J., Gaverick, S., \& Abrams, R. A. (2010). When meaning matters, look but don't touch: The effects of posture on reading. Memory \& Cognition, 38(5), 555-562. doi: 10.3758/MC.38.5.555

de Monasterio, F. M. (1978). Properties of concentrically organized X and Y ganglion cells of macaque retina. Journal of Neurophysiology, 41(6), 1394-1417.

Denison, R. N., Vu, A. T., Yacoub, E., Feinberg, D. A., \& Silver, M. A. (2014). Functional mapping of the magnocellular and parvocellular subdivisions of human LGN. NeuroImage, 2, 358-369. doi: 10.1016/j.neuroimage.2014.07.019

Derrington, A. M., \& Lennie, P. (1984). Spatial and temporal contrast sensitivities of neurones in the lateral geniculate nucleus of the macaque. Journal of Physiology, 357, 219-240.

Desimone, R., \& Duncan, J. (1995). Neural mechanisms of selective visual attention. Annual Review of Neuroscience, 18, 193-222. doi: 10.1146/annurev.ne.18.030195.001205

Di Lollo, V., Enns, J. T., \& Rensink, R. A. (2000). Competition for consciousness among visual events: The psychophysics of reentrant visual processes. Journal of Experimental Psychology: General, 129(4), 481-507. doi: 10.1037/00963445.129.4.481

Dreher, B., Fukada, Y., \& Rodieck, R. W. (1976). Identification, classification and anatomical segregation of cells with X-like and Y-like properties in the lateral geniculate nucleus of old-world primates. The Journal of Physiology, 258(2), 433452.

Duncan, J., Ward, J., \& Shapiro, K. L. (1994). Direct measurement of attentional dwell time in human vision. Nature, 369, 313-315. 
Dux, P. E., \& Marois, R. (2009). How humans search for targets through time: A review of data and theory from the attentional blink. Attention, Perception, \& Psychophysics, 71(8), 1683-1700. doi: 10.3758/APP.71.8.1683

Enns, J. T., Brehaut, J. C., \& Shore, D. I. (1999). The duration of a brief event in the mind's eye. Journal of General Psychology, 126(4), 355-372. doi:

$10.1080 / 00221309909595371$

Enns, J. T., \& Di Lollo, V. (1997). Object substitution: A new form of masking in unattended visual locations. Psychological Science, 8(2), 135-139. doi: 10.1111/j.14679280.1997.tb00696.x

Eriksen, C. W., \& Hoffman, J. E. (1972). Some characteristics of selective attention in visual perception determined by vocal reaction time. Perception \& Psychophysics, 11(2), 169-171. doi: 10.3758/BF03210367

Eriksen, C. W., \& St. James, J. D. (1986). Visual attention within and around the field of focal attention: A zoom lens model. Perception \& Psychophysics, 40(4), 225-240. doi: 10.3758/BF03211502

Eriksen, C. W., \& Yeh, Y.-Y. (1985). Allocation of attention in the visual field. Journal of Experimental Psychology: Human Perception and Performance, 11(5), 583-597. doi: 10.1037/0096-1523.11.5.583

Festman, Y., Adam, J. J., Pratt, J., \& Fischer, M. H. (2013a). Both hand position and movement direction modulate visual attention. Frontiers in Psychology, 4, 657. doi: 10.3389/fpsyg.2013.00657

Festman, Y., Adam, J. J., Pratt, J., \& Fischer, M. H. (2013b). Continuous hand movement induces a far-hand bias in attentional priority. Attention, Perception, \& Psychophysics, 75(4), 644-649. doi: 10.3758/s13414-013-0430-4 
Filmer, H. L., Mattingley, J. B., \& Dux, P. E. (2014). Size (mostly) doesn't matter: The role of set size in object substitution masking. Attention, Perception, \& Psychophysics. doi: 10.3758/s13414-014-0692-5

Folk, C. L., Remington, R. W., \& Johnston, J. C. (1992). Involuntary covert orienting is contingent on attentional control settings. Journal of Experimental Psychology: Human Perception \& Performance, 18(4), 1030-1044. doi: 10.1037/00961523.18.4.1030

Ganel, T., \& Goodale, M. A. (2003). Visual control of action but not perception requires analytical processing of object shape. [Letter]. Nature, 426(6967), 664-667. doi: 10.1038/nature02156

Garza, J. P., Strom, M. J., Wright, C. E., Roberts, R. J., \& Reed, C. (2013). Top-down influences mediate hand bias in spatial attention. Attention, Perception \& Psychophysics, 75, 819-823. doi: 10.3758/s13414-013-0480-7

Gauthier, I., Tarr, M. J., Anderson, A. W., Skudlarski, P., \& Gore, J. C. (1999). Activation of the middle fusiform 'face area' increases with expertise in recognizing novel objects. Nature Neuroscience, 2(6), 568-573. doi: 10.1038/9224

Goodale, M. A., \& Milner, A. D. (1992). Separate visual pathways for perception and action. Trends in Neurosciences, 15(1), 20-25. doi: 10.1016/0166-2236\%2892\%2990344-8

Goodale, M. A., \& Westwood, D. A. (2004). An evolving view of duplex vision: Separate but interacting cortical pathways for perception and action. Current Opinion in Neurobiology, 14(2), 203-211. doi: 10.1016/j.conb.2004.03.002

Goodhew, S. C., Boal, H. L., \& Edwards, M. (2014). A magnocellular contribution to conscious perception via temporal object segmentation. Journal of Experimental Psychology: Human Perception and Performance, 40(3), 948-959. doi: 10.1037/a0035769 
Goodhew, S. C., \& Clarke, R. (revision under review). Contributions of parvocellular and magnocellular pathways to visual perception near the hands are not fixed, but can be dynamically altered.

Goodhew, S. C., Edwards, M., Boal, H. L., \& Bell, J. (2015). Two objects or one? Similarity rather than complexity determines objecthood when resolving dynamic input. Journal of Experimental Psychology: Human Perception and Performance, 41(1), 102-110. doi: $10.1037 / x h p 0000022$

Goodhew, S. C., Fogel, N., \& Pratt, J. (2014). The nature of altered vision near the hands: Evidence for the magnocellular enhancement account from object correspondence through occlusion. Psychonomic Bulletin \& Review, 21(6), 1452-1458. doi: $10.3758 / \mathrm{s} 13423-014-0622-5$

Goodhew, S. C., Gozli, D. G., Ferber, S., \& Pratt, J. (2013). Reduced temporal fusion in nearhand space. Psychological Science, 24(6), 891-900. doi: 10.1177/0956797612463402

Goodhew, S. C., Kendall, W., Ferber, S., \& Pratt, J. (2014). Setting semantics: Conceptual set can determine the physical properties that capture attention. Attention, Perception, \& Psychophysics, 76(6), 1577-1589. doi: 10.3758/s13414-014-0686-3

Goodhew, S. C., Pratt, J., Dux, P. E., \& Ferber, S. (2013). Substituting objects from consciousness: A review of object substitution masking. Psychonomic Bulletin \& Review, 20(5), 859-877. doi: 10.3758/s13423-013-0400-9

Gozli, D. G., Ardron, J., \& Pratt, J. (2014). Reduced visual feature binding in the near-hand space. Attention, Perception, \& Psychophysics Apr(Pagination), No Pagination Specified. doi: 10.3758/s13414-014-0673-8

Gozli, D. G., West, G. L., \& Pratt, J. (2012). Hand position alters vision by biasing processing through different visual pathways. Cognition, 124(2), 244-250. doi: 10.1016/j.cognition.2012.04.008 
Graziano, M., Yap, G., \& Gross, C. (1994). Coding of visual space by premotor neurons. Science, 266(5187), 1054-1057. doi: 10.1126/science.7973661

Greenwood, P., \& Parasuraman, R. (1999). Scale of attentional focus in visual search. Perception \& Psychophysics, 61(5), 837-859. doi: 10.3758/BF03206901

Greenwood, P., \& Parasuraman, R. (2004). The scaling of spatial attention in visual search and its modification in healthy aging. Perception \& Psychophysics, 66(1), 3-22. doi: 10.3758/BF03194857

Grill-Spector, K., Kourtzi, Z., \& Kanwisher, N. (2001). The lateral occipital complex and its role in object recognition. Vision Research, 41(10-11), 1409-1422. doi: 10.1016/S0042-6989(01)00073-6

Grill-Spector, K., Tammar, K., Hendler, T., \& Malach, R. (2000). The dynamics of objectselective activation correlate with recognition performance in humans. Nature Neuroscience, 3, 837-843.

Grubert, A., Righi, L. L., \& Eimer, M. (2013). A unitary focus of spatial attention during attentional capture: Evidence from event-related brain potentials. Journal of Vision, 13(3), 9.

Hollingworth, A., \& Franconeri, S. L. (2009). Object correspondence across brief occlusion is established on the basis of both spatiotemporal and surface feature cues. Cognition, 113(2), 150-166. doi: 10.1016/j.cognition.2009.08.004

Hubner, R. (1997). The effect of spatial frequency on global precedence and hemispheric differences. Perception \& Psychophysics, 59(2), 187-201. doi: 10.3758/BF03211888

Jolicoeur, P. (1998). Modulation of the attentional blink by on-line response selection: Evidence from speeded and unspeeded task1 decisions. Memory \& Cognition, 26, 1014-1032. 
Jonides, J., \& Yantis, S. (1988). Uniqueness of abrupt visual onset in capturing attention. Perception \& Psychophysics, 43(4), 346-354. doi: 10.3758/BF03208805

Kahneman, D., Treisman, A., \& Gibbs, B. J. (1992). The reviewing of object files: Objectspecific integration of information. Cognitive Psychology, 24(2), 175-219. doi: 10.1016/0010-0285\%2892\%2990007-O

Kanwisher, N., \& Yovel, G. (2006). The fusiform face area: A cortical region specialized for the perception of faces. Philosophical Transactions of the Royal Society B, 361, 21092128.

Kastner, S., \& Pinsk, M. A. (2004). Visual attention as a multilevel selection process. Cognitive, Affective, \& Behavioral Neuroscience, 4(4), 483-500. doi:

\subsection{8/CABN.4.4.483}

Kelly, S. P., \& Brockmole, J. R. (2014). Hand proximity differentially affects visual working memory for color and orientation in a binding task. Front Psychol, 5(318), 318. doi: 10.3389/fpsyg.2014.00318

Kennett, S., Spence, C., \& Driver, J. (2002). Visuo-tactile links in covert exogenous spatial attention remap across changes in unseen hand posture. Perception \& Psychophysics, 64(7), 1083-1094. doi: 10.3758/BF03194758

Klein, R. M. (2000). Inhibition of return. Trends in Cognitive Sciences, 4(4), 138-147. doi: 10.1016/S1364-6613(00)01452-2

Kveraga, K., Boshyan, J., \& Bar, M. (2007). Magnocellular projections as the trigger of topdown facilitation in recognition. Journal of Neuroscience, 27(48), 13232-13240. doi: 10.1523/jneurosci.3481-07.2007

LaBerge, D. (1983). Spatial extent of attention to letters and words. Journal of Experimental Psychology: Human Perception and Performance, 9(3), 371-379. doi: 10.1037/00961523.9.3.371 
Legge, G. E. (1978). Sustained and transient mechanisms in human vision: Temporal and spatial properties. Vision Research, 18(1), 69-81. doi: 10.1016/00426989\%2878\%2990079-2

Livingstone, M., \& Hubel, D. (1988). Segregation of form, color, movement, and depth: Anatomy, physiology, and perception. Science, 240(4853), 740-749. doi: 10.1126/science.3283936

Lleras, A., \& Moore, C. M. (2003). When the target becomes the mask: Using apparent motion to isolate the object-level component of object substitution masking. Journal of Experimental Psychology: Human Perception and Performance, 29(1), 106-120. doi: 10.1037/0096-1523.29.1.106

Luck, S. J., \& Vogel, E. K. (1997). The capacity of visual working memory for features and conjunctions. Nature, 390(6657), 279-281. doi: 10.1038/36846

Luiga, I., \& Bachmann, T. (2008). Luminance processing in object substitution masking. Vision Research, 48(7), 937-945. doi: 10.1016/j.visres.2008.01.001

Maunsell, J. H., Nealey, T. A., \& DePriest, D. D. (1990). Magnocellular and parvocellular contributions to responses in the middle temporal visual area (MT) of the macaque monkey. Journal of Neuroscience, 10(10), 3323-3334.

Merigan, W. H., \& Maunsell, J. H. (1993). How parallel are the primate visual pathways? Annual Review of Neuroscience, 16, 369-402. doi: 10.1146/annurev.ne.16.030193.002101

Mishkin, M., \& Ungerleider, L. G. (1982). Contribution of striate inputs to the visuospatial functions of parieto-preoccipital cortex in monkeys. Behavioural Brain Research, 6(1), 57-77. doi: 10.1016/0166-4328\%2882\%2990081-X 
Mishkin, M., Ungerleider, L. G., \& Macko, K. A. (1983). Object vision and spatial vision: Two cortical pathways. Trends in Neurosciences, 6(10), 414-417. doi: 10.1016/01662236\%2883\%2990190-X

Moore, C. M., \& Lleras, A. (2005). On the role of object representations in substitution masking. Journal of Experimental Psychology: Human Perception and Performance, 31(6), 1171-1180. doi: 10.1037/0096-1523.31.6.1171

Most, S. B., Simons, D. J., Scholl, B. J., Jimenez, R., Clifford, E., \& Chabris, C. F. (2001). How not to be seen: The contribution of similarity and selective ignoring to sustained inattentional blindness. Psychological Science, 12(1), 9-17. doi: 10.1111/14679280.00303

Muller, N. G., Bartelt, O. A., Donner, T. H., Villringer, A., \& Brandt, S. A. (2003). A physiological correlate of the "zoom lens" of visual attention. Journal of Neuroscience, 23(9), 3561-3565.

Nassi, J. J., \& Callaway, E. M. (2007). Specialized circuits from primary visual cortex to V2 and area MT. Neuron, 55(5), 799-808. doi: 10.1016/j.neuron.2007.07.037

O'Craven, K. M., Downing, P. E., \& Kanwisher, N. (1999). fMRI evidence for objects as the units of attentional selection. Nature, 401, 584-587.

Park, G. D., Strom, M., \& Reed, C. L. (2013). To the end! Distribution of attention along a tool in peri- and extrapersonal space. Experimental Brain Research, 227(4), 423-432. doi: 10.1007/s00221-013-3439-y

Pilling, M., Gellatly, A., Argyropoulos, Y., \& Skarratt, P. (2014). Exogenous spatial precuing reliably modulates object processing but not object substitution masking. Attention, Perception, \& Psychophysics, 76(6), 1560-1576. doi: 10.3758/s13414-014-0661-z

Popper, K. (1959). The logic of scientific discovery. London: Hutchinson \& Co. 
Posner, M. I. (1980). Orienting of attention. The Quarterly Journal of Experimental Psychology, 32(1), 3-25. doi: 10.1080/00335558008248231

Posner, M. I., \& Cohen, Y. (1984). Components of visual orienting. In H. Bouma \& D. Bouwhuis (Eds.), Attention \& Performance X (pp. 531-556). Hillsdale: Erlbaum.

Posner, M. I., Rafal, R. D., Choate, L. S., \& Vaughan, J. (1985). Inhibition of return: Neural basis and function. Cognitive Neuropsychology, 2(3), 211-228. doi: $10.1080 / 02643298508252866$

Posner, M. I., Walker, J. A., Friedrich, F. A., \& Rafal, R. D. (1987). How do the parietal lobes direct covert attention? Neuropsychologia, 25(1-A), 135-145. doi: 10.1016/0028-3932\%2887\%2990049-2

Previc, F. H. (1990). Functional specialization in the lower and upper visual fields in humans: Its ecological origins and neurophsyiological implications. Behavioral and Brain Sciences, 13(3). doi: 10.1017/S0140525X00080018

Raymond, J. E., Shapiro, K. L., \& Arnell, K. M. (1992). Temporary suppression of visual processing in an RSVP task: An attentional blink? Journal of Experimental Psychology: Human Perception and Performance, 18(3), 849-860.

Reed, C., Betz, R., Garza, J. P., \& Roberts, R. J. (2010). Grab it! Biased attention in functional hand and tool space. Attention, Perception, and Psychophysics, 72(1), 236245. doi: 10.3758/APP.72.1.236

Reed, C., Grubb, J., \& Steele, C. (2006). Hands up: Attentional prioritization of space near the hand. Journal of Experimental Psychology: Human Perception and Performance, 32(1), 166-177. doi: 10.1037/0096-1523.32.1.166

Reed, C., Leland, D. S., Brekke, B., \& Hartley, A. A. (2013). Attention's grasp: Early and late hand proximity effects on visual evoked potentials. Frontiers in Psychology, 4, 1-12. 
Schendel, K., \& Robertson, L. C. (2004). Reaching out to see: Arm position can attenuate human visual loss. Journal of Cognitive Neuroscience, 16(6), 935-943. doi: $10.1162 / 0898929041502698$

Schiller, P. H., \& Logothetis, N. K. (1990). The color-opponent and broad-band channels of the primate visual system. Trends in Neurosciences, 13(10), 392-398. doi: 10.1016/0166-2236(90)90117-s

Shulman, G. L., Sullivan, M. A., Gish, K., \& Sakoda, W. J. (1986). The role of spatialfrequency channels in the perception of local and global structure. Perception, 15(3), 259-273. doi: 10.1068/p150259

Spence, C., Nicholls, M. E., Gillespie, N., \& Driver, J. (1998). Cross-modal links in exogenous covert spatial orienting between touch, audition, and vision. Perception \& Psychophysics, 60(4), 544-557. doi: 10.3758/BF03206045

Stroop, J. R. (1935). Studies of interference in serial verbal reactions. Journal of Experimental Psychology, 18(6), 643-662. doi: 10.1037/h0054651

Treisman, A. M., \& Gelade, G. (1980). A feature-integration theory of attention. Cognitive Psychology, 12(1), 97-136. doi: 10.1016/0010-0285(80)90005-5

Tsal, Y., \& Lavie, N. (1988). Attending to color and shape: The special role of location in selective visual processing. Perception \& Psychophysics, 44(1), 15-21. doi: 10.3758/bf03207469

Tseng, P., \& Bridgeman, B. (2011). Improved change detection with nearby hands. Experimental Brain Research, 209(2), 257-269. doi: DOI 10.1007/s00221-011-2544-z

Vidyasagar, T. R. (1999). A neuronal model of attentional spotlight: Parietal guiding the temporal. Brain Research Reviews, 30, 66-76. 
Visser, T. A. W. (2007). Masking T1 difficulty: Processing time and the attentional blink. Journal of Experimental Psychology: Human Perception and Performance, 33, 285297.

Weidler, B. J., \& Abrams, R. A. (2013). Hand proximity - not arm posture - alters vision near the hands. Attention Perception \& Psychophysics. doi: 10.3758/s13414-013-0456-7

Wiesel, T. N., \& Hubel, D. H. (1966). Spatial and chromatic interactions in lateral geniculate body of rhesus monkey. Journal of Neurophysiology, 29(6), 1115-1156.

Wyble, B., Folk, C., \& Potter, M. (2013). Contingent attentional capture by conceptually relevant images. Journal of Experimental Psychology: Human Perception and Performance, 39(3), 861-871. doi: 10.1037/a0030517

Yantis, S., \& Jonides, J. (1984). Abrupt visual onsets and selective attention: Evidence from visual search. Journal of Experimental Psychology: Human Perception and Performance, 10(5), 601-621. doi: 10.1037/0096-1523.10.5.601

Yeshurun, Y. (2004). Isoluminant stimuli and red background attenuate the effects of transient spatial attention on temporal resolution. Vision Research, 44(12), 13751387. doi: 10.1016/j.visres.2003.12.016

Yeshurun, Y., \& Carrasco, M. (1998). Attention improves or impairs visual performance by enhancing spatial resolution. Nature, 396(6706), 72-75. doi: 10.1038/23936

Yeshurun, Y., \& Hein, E. (2011). Transient attention degrades perceived apparent motion. Perception, 40(8), 905-918. doi: 10.1068/p7016

Yeshurun, Y., \& Levy, L. (2003). Transient spatial attention degrades temporal resolution. Psychological Science, 14(3), 225-231. doi: 10.1111/1467-9280.02436

Yeshurun, Y., \& Marom, G. (2008). Transient spatial attention and the perceived duration of brief visual events. Visual Cognition, 16(6), 826-848. doi:

$10.1080 / 13506280701588022$ 
Yeshurun, Y., \& Rashal, E. (2010). Preceuing attention to the target location diminishes crowding and reduces the critical distance. Journal of Vision, 10(10), 1-12. doi: $10.1167 / 10.10 .16$

Yeshurun, Y., \& Sabo, G. (2012). Differential effects of transient attention on inferred parvocellular and magnocellular processing. Vision Research, 74, 21-29. doi: 10.1016/j.visres.2012.06.006 